\title{
The Role of Ets Factors in Tumor Angiogenesis
}

\author{
Peter Oettgen \\ Division of Cardiology, and Molecular and Vascular Biology, Department of Medicine, Research North RM 270D, \\ Beth Israel Deaconess Medical Center, 330 Brookline Avenue, Boston, MA 02115, USA
}

Correspondence should be addressed to Peter Oettgen, joettgen@bidmc.harvard.edu

Received 20 October 2009; Revised 6 February 2010; Accepted 2 March 2010

Academic Editor: Debabrata Mukhopadhyay

Copyright () 2010 Peter Oettgen. This is an open access article distributed under the Creative Commons Attribution License, which permits unrestricted use, distribution, and reproduction in any medium, provided the original work is properly cited.

\begin{abstract}
Angiogenesis is a critical component of tumor growth. A number of growth factors, including VEGF, FGF, and HGF, have been implicated as angiogenic growth factors that promote tumor angiogenesis in different types of cancer. Ets-1 is the prototypic member of the Ets transcription factor family. Ets-1 is known to be a downstream mediator of angiogenic growth factors. Expression of Ets-1 in a variety of different tumors is associated with increased angiogenesis. A role for other selected members of the Ets transcription factor family has also been shown to be important for the development of tumor angiogenesis. Because Ets factors also express a number of other important genes involved in cell growth, they contribute not only to tumor growth, but to disease progression. Targeting Ets factors in mouse tumor models through the use of dominant-negative Ets proteins or membrane permeable peptides directed at competitively inhibiting the DNA binding domain has now demonstrated the therapeutic potential of inhibiting selected Ets transcription factors to limit tumor growth and disease progression.
\end{abstract}

Ets-1 is the prototypic member of the Ets transcription factor family [1]. Several studies have demonstrated a role for Ets transcription factors in the regulation of endothelialspecific genes including VEGF-R1, VEGF-R2, Tie1, and Tie2. Whereas, the Ets factors Ets- 1 and Ets-2 potently transactivate the Flt-1 gene promoter, they do not appear to regulate the Tie1 or Tie2 gene promoters $[2,3]$. Ets- 1 has been shown to cooperate with HIF- $2 \alpha$ in the setting of hypoxia to regulate the expression of the VEGF-R2 $[4,5]$. In contrast, the Ets factor ELF-1 is a potent transactivator of the Tie1 and Tie2 genes $[2,3]$. ELF-1 has been shown to regulate other genes involved in angiogenesis including angiopoietin-2 and endothelial nitric oxide synthase $[6,7]$. Ets-2 regulates the expression of CD13/aminopeptidase N (APN) in human endothelial cells [8]. Knockdown of Ets2 in human endothelial cells using siRNA oligonucleotides significantly reduced the ability of the endothelial cells to form tubes and capillary networks. The selectivity of the different Ets factors to transactivate different target genes also correlates with their ability to bind to specific conserved Ets binding sites within these genes. However, when the DNA binding domain is highly homologous, as is the case for Ets-1 and Ets-2, there may be a significant overlap with respect to their downstream target genes.
Ets factors can be subcategorized into subfamilies based on DNA and protein sequence homology. For example, Elf-1, Nerf-2, and Mef are highly related and belong to one subfamily. Similarly Fli1 and ERG constitute another subfamily, as do Ets1 and Ets2. The subfamily members often have overlapping functions [9]. From an evolutionary standpoint, this built-in redundancy may be protective against the effects of genetic mutations of the individual family members for critical developmental processes such as vasculogenesis. The DNA binding domain of the Ets family of proteins is a highly conserved region of approximately 85 residues that shares a strong sequence homology and threedimensional structural scaffold that closely mimics the helixturn-helix family of DNA binding proteins $[10,11]$. Previous investigations have clearly established that the several highly conserved residues that are localized within the recognition helix, H3, are responsible for anchoring the Ets domain through requisite DNA contacts within the major groove. However, residues within the turns separating helices $\mathrm{H} 2$ and $\mathrm{H} 3$ and the first two $\beta$-strands are also involved in critical phosphate backbone contacts within the DNA minor groove.

Expressions of selected Ets factors are also enriched at sites of active blood vessel development during embryogenesis. The Ets transcription factor Fli-1 is enriched in 
the developing blood vessels of zebrafish embryos [12]. ELF1 is highly expressed in extra-embryonic and embryonic blood vessels of the developing mouse and chicken embryos $[7,13]$. The Ets factor Ets- 1 is also enriched in the developing blood vessels of the chicken, and antisense oligonucleotides have been shown to inhibit angiogenesis when delivered to the chicken chorioallantoic membrane [14]. Homozygous inactivation of Ets1 is associated with abnormalities in T cell function, but with no defects in vascular development or angiogenesis [15]. Similarly, while Ets2 is a critical regulator of trophoblast function during extraembryonic development it is dispensable for development of the embryo proper [16]. Mice with a homozygous hypomorphic mutant of Ets2, in which the conserved threonine 72 phosphorylation site is mutated, and Ets2 ${ }^{\mathrm{T} 72 \mathrm{~A} / \mathrm{T} 72 \mathrm{~A}}$ mice are viable and appear normal [17]. When both Ets-1 and Ets-2 were simultaneously targeted, this led to striking defects in vascular development [18].

In addition to their role in regulating endothelial cell restricted genes, Ets factors have also been shown to function upstream and downstream of a number of angiogenic growth factors [19]. Overexpression of Ets-1 in tissues is associated with increased expression of both vascular endothelial growth factor (VEGF) and hepatocyte growth factor (HGF). Neutralizing antibodies to VEGF and HGF markedly attenuate the angiogenic effects of overexpressing Ets-1. Ets-1 regulates the expression of several downstream targets in endothelial cells that promote an angiogenic phenotype including the VEGF receptors (VEGF-R1 and VEGF-R2), urokinase, and several matrix metalloproteinase's (MMPs) including MMP-1, MMP-3, MMP-9 (Figure 1). Interestingly, HGF is also capable of inducing the expression of Ets-1, thereby creating a positive feedback loop at sites where either Ets-1 or HGF is expressed. In addition to HGF, fibroblast growth factor (FGF) and VEGF gene expression are regulated by Ets-1 [20, 21]. More recently, the chemokine CCL2 (MCP-1/JE) has been shown to promote angiogenesis and one of the main downstream effectors is Ets-1 [22]. The particular downstream targets identified in response to CCL2 in endothelial cells are the $\beta 3$ integrins. Ets- 1 is also involved in the regulation of CCL2 expression [23].

Several studies support a role for Ets-1 expression in the development of tumor angiogenesis in many different types of human cancer. Increased expression of Ets-1 in tumors is often associated with a worse prognosis (summarized in Table 1). In ovarian cancer, for example, Ets-1 expression strongly correlates with the degree of angiogenesis in the primary tumor and the development of metastatic lesions [24]. The prognosis is also significantly worse in those patients with high levels of Ets-1 expression in the tissues derived from the primary cancer. The survival rate of patients with histologically demonstrated high levels of Ets-1 expression at 24 months was 30\% whereas that of patients with low levels was 70\% [25]. These statistics support the overall concept that Ets-1 expression in ovarian cancers contributes to tumor growth and progression that is at least in part mediated by an increase in the degree of angiogenesis. Additional factors promoting tumor growth and progression are discussed below.
Esophageal squamous cell carcinoma (ESCC) is associated with a late presentation due to limited symptoms early in the disease. However, once detected, ESCC is a highly aggressive tumor with a propensity to metastasize. Ets-1 is overexpressed in 44/55 (80\%) of tumor tissues obtained from patients with ESCC [26]. VEGF expression was observed in most of the Ets-1 overexpressing tissues and was strongly correlated with lymphnode metastasis. KaplanMeier survival analysis for patients with concomitant tissue expression of Ets-1 and VEGF demonstrated a significantly worse disease free survival. This again supports the concept that increased angiogenesis in Ets-1 expressing tumors is associated with increased tumor progression and a worse prognosis.

VEGF and Ets- 1 are also highly expressed in breast carcinoma [27]. Ets-1 is expressed in 53\% of tumors of patients with newly diagnosed breast cancer. Ets-1 overexpression in breast cancer is associated with more invasive tumors and a significantly poorer prognosis [28]. At 5 years, less than $20 \%$ of patients with Ets-1 negative tumors have relapsed, compared to nearly $50 \%$ of those with Ets- 1 expressing tumors. The expression of Ets-1 strongly correlated with the microvessel density within the tumors and lymph node involvement. In a study of 123 patients with primary breast cancer followed for 62 months, Ets- 1 expression correlated strongly with VEGF expression and was an independent predictor of poor prognosis [28]. As a result of these findings, antiangiogenic therapy is currently being evaluated as adjunctive therapy for breast cancer.

In a study of 60 patients with uterine cancer a strong Ets- 1 expression was observed in $66 \%$ of patients, and was associated with increased tumor microvessel density [34]. In patients with cervical carcinoma, high Ets-1 expression was observed in tissues obtained from 25/60 (42\%) patients and was also associated with a high microvessels density [30]. Furthermore, the 24-month survival rate of patients with high ets- 1 expression was significantly worse at $54 \%$ compared to $92 \%$ for those patients with low expression of Ets-1 in the tumors. These results also support a role for Ets1 in tumor progression that is at least in part mediated by increased angiogenesis.

Ets-1 expression has also been shown to correlate with higher microvessel density and a worse prognosis in patients with gastric cancer [31]. Of the 102 patients with primary gastric carcinoma, not involving invasion into the muscularis propria or subserosa, Ets-1 expression was observed in $76 \%$ of patients. The 5-year survival rate for these patients was $67 \%$ for patients with Ets-1 positive tumors and $89 \%$ for those with Ets-1 negative tumors. Ets-1 expression has also been evaluated in patients with colorectal carcinoma [32]. High Ets- 1 expression was observed in $48.4 \%$ of patients and correlated strongly with increased microvessel density and the expression of VEGF. Similar to the studies in uterine cancer, this study also supports a role for expression of Ets1 in cancer progression that is at least in part mediated by increased angiogenesis.

The role of Ets-1 in human brain cancer has also been evaluated. Ets- 1 expression was evaluated in 81 primary and 20 recurrent astrocytic tumors [33]. 65\% of glioblastomas 
TABLE 1: Summary of Ets-1 expression, microvessel density (MVD), and prognosis in human cancer.

\begin{tabular}{|c|c|c|c|c|}
\hline Tumor type & Number & \% Ets-1 positive* & MVD/VEGF & Prognosis** \\
\hline \multirow[t]{2}{*}{ (1) Ovarian [24] } & 30 & 66 & increased MVD & 2 years survival: \\
\hline & & & & $10 \%$ Ets- $1(+) ; 60 \%$ Ets- $1(-)$ \\
\hline \multirow[t]{2}{*}{ (2) Ovarian [25] } & 30 & 50 & increased MVD & 2 years survival: \\
\hline & & & & $30 \%$ Ets-1 $(+) ; 70 \%$ Ets-1 $(-)$ \\
\hline (3) Esophageal [26] & 55 & 80 & increased MVD & not evaluated \\
\hline (4) Breast [27] & 48 & 71 & increased MVD & not evaluated \\
\hline \multirow[t]{2}{*}{ (5) Breast [28] } & 123 & 62 & not evaluated & 5 years relapse free survival: \\
\hline & & & & $55 \%$ Ets- $1(+) ; 85 \%$ Ets-1 $(-)$ \\
\hline (6) Endometrial [29] & 60 & 66 & increased VEGF & not evaluated \\
\hline \multirow[t]{2}{*}{ (7) Cervical [30] } & 60 & 42 & increased MVD & 2 years survival: \\
\hline & & & & $54 \%$ Ets-1 $(+) ; 92 \%$ Ets-1 $(-)$ \\
\hline \multirow[t]{2}{*}{ (8) Gastric [31] } & 102 & 76 & increased MVD & 5 years survival: \\
\hline & & & & $67 \%$ Ets- $1(+) ; 89 \%$ Ets- $1(-)$ \\
\hline (9) Colon [32] & 95 & 48 & increased MVD & not evaluated \\
\hline (10) Brain [33] & 101 & 32 & not evaluated & not evaluated \\
\hline
\end{tabular}

"Number" refers to number of patients in the study.

*\% refers to percent of primary tumors that express Ets- 1 .

$* * \%$ refers to percent of patients that are relapse free at different time points with primary tumors that expressed Ets-1 (+) or did not express Ets-1 ( $)$.

"MVD/VEGF" refers to evaluation of either VEGF expression or microvascular density (MVD) in the tumors

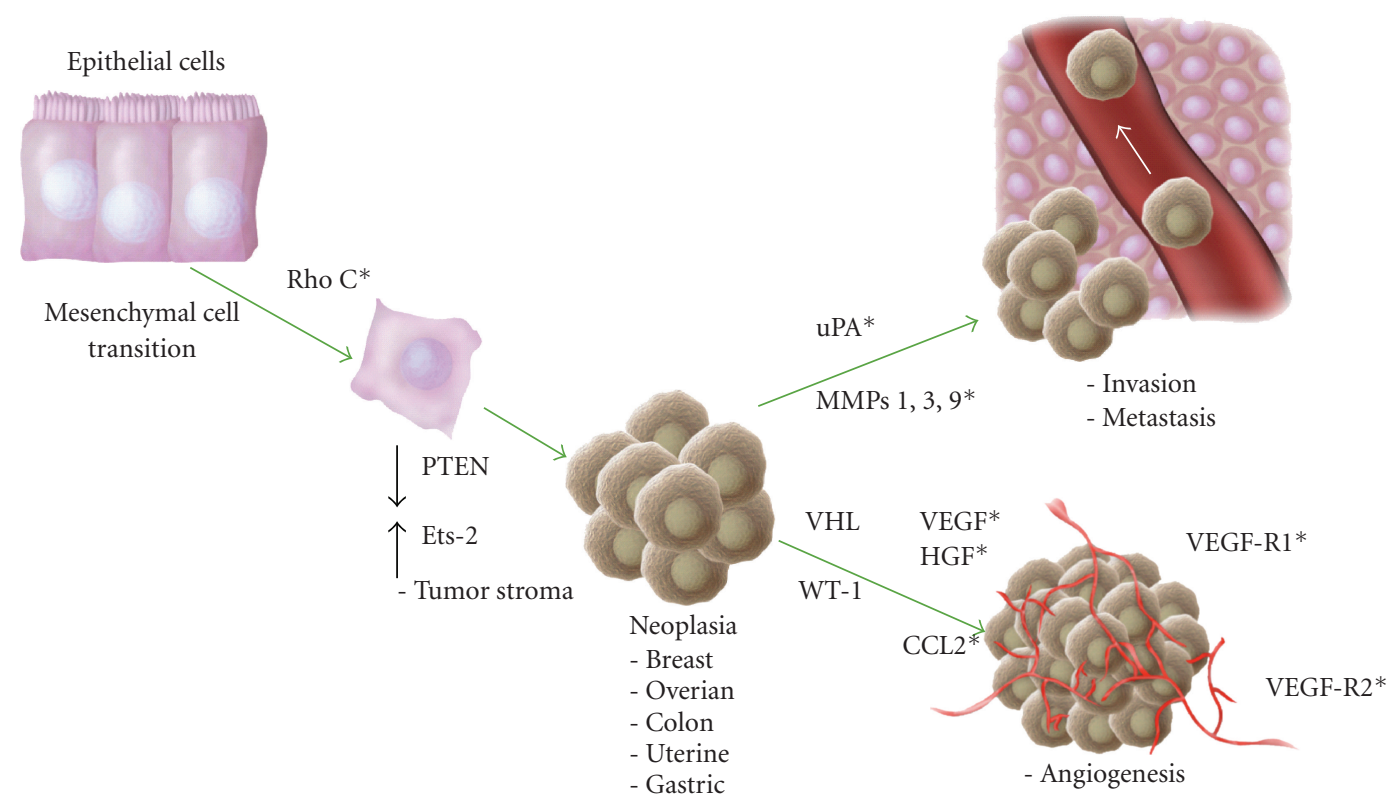

Figure 1: The role of Ets-1 in tumor development, progression, and angiogenesis. The role of Ets-1 is depicted during different stages of neoplasia from the epithelial-mesenchymal transition (EMT) to tumor growth, invasion, metastasis, and tumor angiogenesis. $(*)$ denotes those genes that are regulated by Ets-1.

(grade IV astrocytomas) stained for Ets-1, 25\% of anaplastic astrocytomas (Grade III) were positive for Ets-1, and none of the low-grade astrocytomas (Grade II) stained positively for Ets-1. Therefore the expression of Ets-1 was significantly associated with the tumor grade. Normal brain tissue did not express significant amounts of Ets- 1 protein. Ets- 1 also appears to play a role in regulating tumor angiogenesis in neuroblastomas [35]. Ets-1 expression is higher in undifferentiated human neuroblastomas. Ets- 1 expression is stimulated by gastrin-releasing peptide (Grp) that stimulates neuroblastoma growth, and the induction of Ets-1 in these cells promotes the expression and secretion of the angiogenic chemokine interleukin-8.

The expressions of Ets factors have also been shown to be up-regulated in a number of hematological malignancies either through translocations or through overexpression. One example of an Ets factor that is involved in several different types of hematological malignancies is Tel (ETV6). 
The hematological malignancies include chronic myeloid leukemia, acute myeloid leukemia, acute lymphoblastic leukemia, and non-Hodgkin's lymphoma [36, 37]. The evidence that angiogenesis plays a pathophysiological role in leukemia has been well documented [38]. Angiogenesis in human leukemias is demonstrated by increased microvessel density (MVD) in the bone marrow, and is associated with the increased expression of hypoxia inducible factor $1 \alpha$ (HIF$1 \alpha$ ), the increased expression of multiple angiogenic factors including VEGF, bFGF, and angiopoetin-2, and a reduction in the expression of endogenous angiogenesis inhibitors such as thrombospondin-1 [39]. Because ETV6 has been shown to be important for yolk sac angiogenesis, its upregulation in several hematological malignancies also suggests that it may likely play an important role in regulating angiogenic factors in hematological malignancies. Furthermore, because several of the Ets factors have been shown to function as downstream effectors of angiogenic factors, targeting ETV6 and other Ets factors in these cancers may not only limit direct tumor cell growth but also may limit the local microenvironment through inhibitory effects on angiogenesis.

With regard to tumor progression, a number of factors have been shown to promote tumor growth and/or progression. Some of these are directly regulated by Ets factors, while others act synergistically with Ets to regulate specific target genes. One of the earliest events in the development of tumors from epithelial cells is the so-called epithelial to mesenchymal transition (EMT). This process has been associated with increased expression of RhoC, loss of Ecadherin, and acquisition of mesenchymal characteristics. It has recently been shown that the expression of RhoC is regulated by Ets-1 [40]. The tumor stroma is believed to be an important contributor to some of the most malignant characteristics of epithelial tumors. It has recently been shown that targeted deletion of the tumor suppressor PTEN in stromal fibroblasts of mouse mammary glands accelerates the initiation, progression, and malignant transformation of mammary epithelial tumors [41]. More importantly, it was found that loss of PTEN in the stromal fibroblasts was associated with an increase in the expression of Ets-2. The deletion of Ets-2 and PTEN was associated with a decrease in tumor vasculature and the recruitment of macrophages, resulting in fewer and smaller tumors than deleting PTEN alone. There are other recently described links to certain Ets proteins and the development of tumor angiogenesis. For example, the Wilm's tumor suppressor WT-1 was detected in the endothelium of angiogenic blood vessels of 95\% of 113 tumors of different origins. Ets-1 and WT-1 have overlapping expression patterns. It was shown that silencing of WT-1 in endothelial cells reduced cell proliferation, cell migration, and endothelial tube formation and that silencing of WT1 diminished the expression of Ets-1 in the tumors [42]. Mutations of the tumor suppressor gene von Hippel-Lindau (VHL) are associated with highly vascularized tumors that in part have been linked to stabilization of the transcription factor hypoxia-inducible factor (HIF), which upregulates proangiogenic factors such as vascular endothelial growth factor (VEGF). Down regulation of VHL in endothelial cells has recently been linked to Ets-1 activation via increased
FGF signal transduction [43]. In Kaposi's sarcoma angiogenesis is associated with the induction of angiopoeitin-2 the expression of which is regulated by the transcription factors AP-1 and Ets-1 [44]. Another important gene for the regulation of tumor angiogenesis, wound healing, and fibrosis is connective tissue growth factor (CCN2). Under pathological conditions, such as wound healing or tumor formation it has recently been shown that Ets- 1 competitively inhibits the function of another Ets factor Fli-1 to promote the expression of CCN2, thereby promoting extracellular matrix deposition during cancer [45].

From a therapeutic standpoint, recent studies support important role for Ets factors as critical transcriptional regulators of tumor angiogenesis. Local administration of a dominant-negative form of Ets-1, encoding the DNA binding domain, significantly blocked tumor angiogenesis and tumor growth [46]. Similarly, in a pancreatic mouse cancer model of angiogenesis, the local administration of a dominant negative Ets-1 peptide using and adenoviral vector significantly blunted tumor growth and blood vessel density [47]. By targeting the DNA binding domain, these therapeutic agents very likely target subfamilies of Ets factors, for example, Ets-1 and Ets-2, rather than being entirely selective for a particular Ets factor [7].

Administration of dominant negative Ets proteins has not been successfully used to block tumor growth systemically. Another strategy that has been used to inhibit the function of transcription factors is the use of membrane permeable peptides. For example, a peptide with a membrane permeable sequence and the nuclear localization signal sequence of the transcription factor NF- $\kappa \mathrm{B}$ can block nuclear import of this transcription factor upon activation [48]. The inhibitory function of this peptide, however, was not specific to NF- $\kappa$ B, and also inhibited other transcription factors such as AP-1, NFAT, and STAT1 from entering the nucleus [49]. Another membrane permeable peptide with the ability to interfere with DNA binding of a transcription factor was recently demonstrated using the Tet repressor (TetR) of Escherichia coli synthesized in tandem with a cell membrane transducing peptide. This peptide was able to repress the expression of a tetracycline responsive reporter unit stably transfected into the genome of HeLa cells [50]. Membrane permeable peptides consisting of the terminal portion of the DNA binding domain of the Ets factor Elf- 1 in tandem with the HIV TAT peptide readily cross the cell membrane and enter the nucleus of cells where they are able to block the function of the Ets factor Elf-1. These peptides were successfully used to systemically block the growth of B16 melanoma tumor cells in vivo [7]. Furthermore, the identification of relatively short peptides of 30 to 40 amino acids long that can selectively block the DNA binding of specific transcription factors, or subsets of transcription factors, suggests that this may be a very potent novel approach toward targeting transcription factors as a therapeutic modality to inhibit tumor growth and angiogenesis. Although these peptides have been used successfully to block tumor growth in small animals, there may be certain limitations. First the cost of making these peptides may be prohibitive for use on a much larger scale in man. Second, if the peptides are 
to be used repeatedly, the peptides may be immunogenic and antibodies directed against the peptides may eventually limit their use. With recent advances in drug discovery, the identification and/or development of small molecule inhibitors of subfamilies of Ets factors may be possible in the future by taking advantage of structural similarities among the subfamilies in the DNA binding domain or other functionally conserved regions of the proteins. Although transcription factors have historically been poor drug targets, the advances in computational chemistry may ultimately lead to the identification of selective inhibitors of Ets and other transcription factors.

\section{References}

[1] B. Wasylyk, S. L. Hahn, and A. Giovane, "The Ets family of transcription factors," European Journal of Biochemistry, vol. 211, no. 1-2, pp. 7-18, 1993.

[2] A. Dube, Y. Akbarali, T. N. Sato, T. A. Libermann, and P. Oettgen, "Role of the Ets transcription factors in the regulation of the vascular-specific Tie2 gene," Circulation Research, vol. 84, no. 10, pp. 1177-1185, 1999.

[3] K. Iljin, A. Dube, S. Kontusaari, et al., "Role of Ets factors in the activity and endothelial cell specificity of the mouse Tie gene promoter," FASEB Journal, vol. 13, no. 2, pp. 377-386, 1999.

[4] G. Elvert, A. Kappel, R. Heidenreich, et al., "Cooperative interaction of hypoxia-inducible factor- $2 \alpha$ (HIF- $2 \alpha)$ and Ets- 1 in the transcriptional activation of vascular endothelial growth factor receptor-2 (Flk-1)," The Journal of Biological Chemistry, vol. 278, no. 9, pp. 7520-7530, 2003.

[5] M. Oikawa, M. Abe, H. Kurosawa, W. Hida, K. Shirato, and Y. Sato, "Hypoxia induces transcription factor ETS-1 via the activity of hypoxia-lnducible factor-1," Biochemical and Biophysical Research Communications, vol. 289, no. 1, pp. 3943, 2001.

[6] A. Hegen, S. Koidl, K. Weindel, D. Marme, H. G. Augustin, and U. Fiedler, "Expression of angiopoietin-2 in endothelial cells is controlled by positive and negative regulatory promoter elements," Arteriosclerosis, Thrombosis, and Vascular Biology, vol. 24, no. 10, pp. 1803-1809, 2004.

[7] X. Huang, C. Brown, W. Ni, E. Maynard, A. C. Rigby, and P. Oettgen, "Critical role for the Ets transcription factor ELF-1 in the development of tumor angiogenesis," Blood, vol. 107, no. 8, pp. 3153-3160, 2006.

[8] N. Petrovic, S. V. Bhagwat, W. J. Ratzan, M. C. Ostrowski, and L. H. Shapiro, "CD13/APN transcription is induced by RAS/MAPK-mediated phosphorylation of Ets-2 in activated endothelial cells," The Journal of Biological Chemistry, vol. 278, no. 49, pp. 49358-49368, 2003.

[9] P. Oettgen, "Functional redundancy of Ets1 and Ets2," Blood, vol. 114, no. 5, pp. 934-935, 2009.

[10] C. W. Garvie, J. Hagman, and C. Wolberger, "Structural studies of Ets-1/Pax5 complex formation on DNA," Molecular Cell, vol. 8, no. 6, pp. 1267-1276, 2001.

[11] F. Pio, C. Z. Ni, R. S. Mitchell, et al., "Co-crystallization of an ETS domain (PU.1) in complex with DNA. Engineering the length of both protein and oligonucleotide," The Journal of Biological Chemistry, vol. 270, no. 41, pp. 24258-24263, 1995.

[12] L. A. Brown, A. R. F. Rodaway, T. F. Schilling, et al., "Insights into early vasculogenesis revealed by expression of the ETSdomain transcription factor Fli-1 in wild-type and mutant zebrafish embryos," Mechanisms of Development, vol. 90, no. 2, pp. 237-252, 2000.

[13] A. Dube, S. Thai, J. Gaspar, et al., "ELF-1 is a transcriptional regulator of the Tie2 gene during vascular development," Circulation Research, vol. 88, no. 2, pp. 237-244, 2001.

[14] N. Wernert, A. Stanjek, A. Hugel, and A. Giannis, "Inhibition of angiogenesis on the chicken chorioallantoic membrane by Ets 1 antisense oligodeoxyribonucleotides," Verhandlungen der Deutschen Gesellschaft fur Pathologie, vol. 83, pp. 212-215, 1999.

[15] K. Barton, N. Muthusamy, C. Fischer, et al., "The Ets-1 transcription factor is required for the development of natural killer cells in mice," Immunity, vol. 9, no. 4, pp. 555-563, 1998.

[16] H. Yamamoto, M. L. Flannery, S. Kupriyanov, et al., "Defective trophoblast function in mice with a targeted mutation of Ets2," Genes and Development, vol. 12, no. 9, pp. 1315-1326, 1998.

[17] A. K. Man, L. J. T. Young, J. A. Tynan, et al., "Ets2-dependent stromal regulation of mouse mammary tumors," Molecular and Cellular Biology, vol. 23, no. 23, pp. 8614-8625, 2003.

[18] G. Wei, R. Srinivasan, C. Z. Cantemir-Stone, et al., "Ets1 and Ets 2 are required for endothelial cell survival during embryonic angiogenesis," Blood, vol. 114, no. 5, pp. 11231130, 2009.

[19] N. Hashiya, N. Jo, M. Aoki, et al., "In vivo evidence of angiogenesis induced by transcription factor ets-1: Ets-1 is located upstream of angiogenesis cascade," Circulation, vol. 109, no. 24, pp. 3035-3041, 2004.

[20] G. Kitange, S. Shibata, Y. Tokunaga, et al., "Ets-1 transcription factor-mediated urokinase-type plasminogen activator expression and invasion in glioma cells stimulated by serum and basic fibroblast growth factors," Laboratory Investigation, vol. 79, no. 4, pp. 407-416, 1999.

[21] Z.-Q. Chen, R. J. Fisher, C. W. Riggs, J. S. Rhim, and J. A. Lautenberger, "Inhibition of vascular endothelial growth factor-induced endothelial cell migration by ETS1 antisense oligonucleotides," Cancer Research, vol. 57, no. 10, pp. 20132019, 1997.

[22] S. M. Stamatovic, R. F. Keep, M. Mostarica-Stojkovic, and A. V. Andjelkovic, "CCL2 regulates angiogenesis via activation of Ets-1 transcription factor," Journal of Immunology, vol. 177, no. 4, pp. 2651-2661, 2006.

[23] Y. Zhan, C. Brown, E. Maynard, et al., "Ets-1 is a critical regulator of Ang II-mediated vascular inflammation and remodeling," Journal of Clinical Investigation, vol. 115, no. 9, pp. 2508-2516, 2005.

[24] J. Fujimoto, I. Aoki, H. Toyoki, et al., "Clinical implications of expression of ETS-1 related to angiogenesis in metastatic lesions of ovarian cancers," Oncology, vol. 66, no. 5, pp. 420428, 2004.

[25] S. Khatun, J. Fujimoto, H. Toyoki, and T. Tamaya, "Clinical implications of expression of ETS-1 in relation to angiogenesis in ovarian cancer," Cancer Science, vol. 94, no. 9, pp. 769-773, 2003.

[26] T. Mukherjee, A. Kumar, M. Mathur, T. K. Chattopadhyay, and R. Ralhan, "Ets-1 and VEGF expression correlates with tumor angiogenesis, lymph node metastasis, and patient survival in esophageal squamous cell carcinoma," Journal of Cancer Research and Clinical Oncology, vol. 129, no. 7, pp. 430-436, 2003.

[27] H. Wu, Y. Li, L. Zhang, G. Zhu, and J. Cai, "Expression of vascular endothelial growth factor and E26 transformationspecific-1 in breast carcinoma," Zhonghua Bing Li Xue Za Zhi, vol. 31, pp. 222-226, 2002. 
[28] P. N. Span, P. Manders, J. J. Heuvel, et al., "Expression of the transcription factor Ets-1 is an independent prognostic marker for relapse-free survival in breast cancer," Oncogene, vol. 21, no. 55, pp. 8506-8509, 2002.

[29] J. Fujimoto, I. Aoki, H. Toyoki, S. Khatun, E. Sato, and T. Tamaya, "Expression of ETS-1 related to angiogenesis in uterine endometrium during the menstrual cycle," Journal of Biomedical Science, vol. 10, no. 3, pp. 320-327, 2003.

[30] J. Fujimoto, I. Aoki, H. Toyoki, S. Khatun, and T. Tamaya, "Clinical implications of expression of ETS-1 related to angiogenesis in uterine cervical cancers," Annals of Oncology, vol. 13, no. 10, pp. 1598-1604, 2002.

[31] S. Tsutsumi, H. Kuwano, T. Asao, K. Nagashima, T. Shimura, and E. Mochiki, "Expression of Ets-1 angiogenesis-related protein in gastric cancer," Cancer Letters, vol. 160, no. 1, pp. 45-50, 2000.

[32] K. Tokuhara, Y. Ogata, M. Nakagawa, and K. Shirouzu, "Ets1 expression in vascular endothelial cells as an angiogenic and prognostic factor in colorectal carcinoma," International Surgery, vol. 88, no. 1, pp. 25-33, 2003.

[33] G. Kitange, M. Kishikawa, T. Nakayama, S. Naito, M. Iseki, and S. Shibata, "Expression of the Ets-1 proto-oncogene correlates with malignant potential in human astrocytic tumors," Modern Pathology, vol. 12, no. 6, pp. 618-626, 1999.

[34] J. Fujimoto, I. Aoki, H. Toyoki, S. Khatun, and T. Tamaya, "Clinical implications of expression of ETS-1 related to angiogenesis in uterine endometrial cancers," Annals of Oncology, vol. 13, no. 10, pp. 1605-1611, 2002.

[35] J. Qiao, J. H. Kang, J. Cree, B. M. Evers, and D. H. Chung, "Ets1 transcription factor mediates gastrin-releasing peptideinduced IL-8 regulation in neuroblastoma cells," Neoplasia, vol. 9, no. 3, pp. 184-191, 2007.

[36] I. Wlodarska, R. La Starza, M. Baens, et al., "Fluorescence in situ hybridization characterization of new translocations involving TEL (ETV6) in a wide spectrum of hematologic malignancies," Blood, vol. 91, no. 4, pp. 1399-1406, 1998.

[37] D. W. Sevilla, S. V. Nandula, A. I. Colovai, et al., "Diffuse large B-cell lymphoma with TEL/ETV6 translocation," Human Pathology, vol. 40, no. 4, pp. 588-593, 2009.

[38] T. Kessler, F. Fehrmann, R. Bieker, W. E. Berdel, and R. M. Mesters, "Vascular endothelial growth factor and its receptor as drug targets in hematological malignancies," Current Drug Targets, vol. 8, no. 2, pp. 257-268, 2007.

[39] W. W. Li, M. Hutnik, and G. Gehr, "Antiangiogenesis in haematological malignancies," British Journal of Haematology, vol. 143, no. 5, pp. 622-631, 2008.

[40] D. I. Bellovin, K. J. Simpson, T. Danilov, et al., "Reciprocal regulation of RhoA and RhoC characterizes the EMT and identifies RhoC as a prognostic marker of colon carcinoma," Oncogene, vol. 25, no. 52, pp. 6959-6967, 2006.

[41] A. J. Trimboli, C. Z. Cantemir-Stone, F. Li, et al., "Pten in stromal fibroblasts suppresses mammary epithelial tumours," Nature, vol. 461, no. 7267, pp. 1084-1091, 2009.

[42] N. Wagner, J. F. Michiels, A. Schedl, and K.-D. Wagner, "The Wilms' tumour suppressor WT1 is involved in endothelial cell proliferation and migration: expression in tumour vessels in vivo," Oncogene, vol. 27, no. 26, pp. 3662-3672, 2008.

[43] K. J. Champion, M. Guinea, V. Dammai, and T. Hsu, "Endothelial function of von Hippel-Lindau tumor suppressor gene: control of fibroblast growth factor receptor signaling," Cancer Research, vol. 68, no. 12, pp. 4649-4657, 2008.
[44] F. C. Ye, D. J. Blackbourn, M. Mengel, et al., "Kaposi’s sarcomaassociated herpesvirus promotes angiogenesis by inducing angiopoietin-2 expression via AP-1 and Ets1," Journal of Virology, vol. 81, no. 8, pp. 3980-3991, 2007.

[45] S. S. Nakerakanti, B. Kapanadze, M. Yamasaki, M. Markiewicz, and M. Trojanowska, "Fli1 and Ets1 have distinct roles in connective tissue growth factor/CCN2 gene regulation and induction of the profibrotic gene program," The Journal of Biological Chemistry, vol. 281, no. 35, pp. 25259-25269, 2006.

[46] A. Pourtier-Manzanedo, C. Vercamer, E. Van Belle, V. Mattot, F. Mouquet, and B. Vandenbunder, "Expression of an Ets1 dominant-negative mutant perturbs normal and tumor angiogenesis in a mouse ear model," Oncogene, vol. 22, no. 12, pp. 1795-1806, 2003.

[47] L. P. Lefter, S. Dima, M. Sunamura, et al., "Transcriptional silencing of ETS-1 efficiently suppresses angiogenesis of pancreatic cancer," Cancer Gene Therapy, vol. 16, no. 2, pp. 137-148, 2009.

[48] L. Zhang, T. R. Torgerson, X. Y. Liu, et al., "Preparation of functionally active cell-permeable peptides by single-step ligation of two peptide modules," Proceedings of the National Academy of Sciences of the United States of America, vol. 95, no. 16, pp. 9184-9189, 1998.

[49] T. R. Torgerson, A. D. Colosia, J. P. Donahue, Y. Z. Lin, and J. Hawiger, "Regulation of NF- $\kappa$ B, AP-1, NFAT, and STAT1 nuclear import in $\mathrm{T}$ lymphocytes by noninvasive delivery of peptide carrying the nuclear localization sequence of NF- $\kappa \mathrm{B}$ p501," Journal of Immunology, vol. 161, no. 11, pp. 6084-6092, 1998.

[50] A. Mortlock, W. Low, and A. Crisanti, "Suppression of gene expression by a cell-permeable Tet repressor," Nucleic Acids Research, vol. 31, article e152, 2003. 


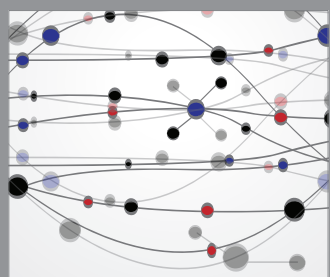

The Scientific World Journal
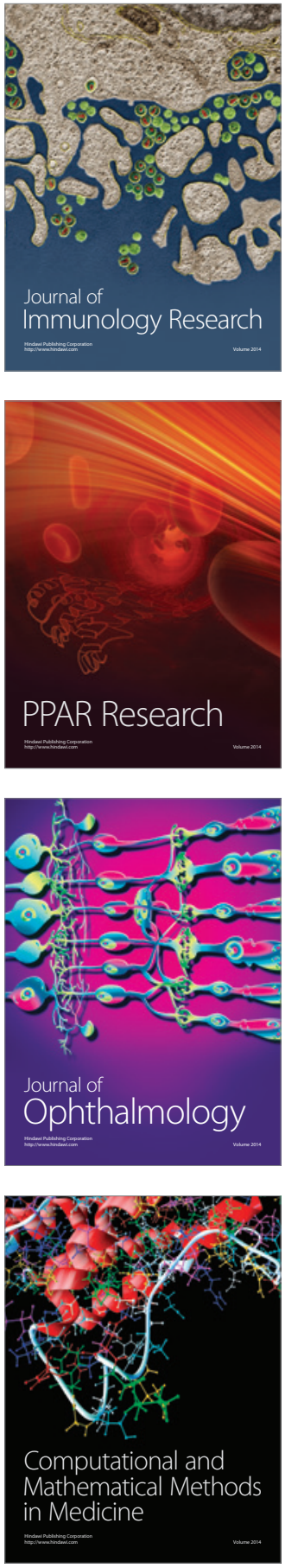

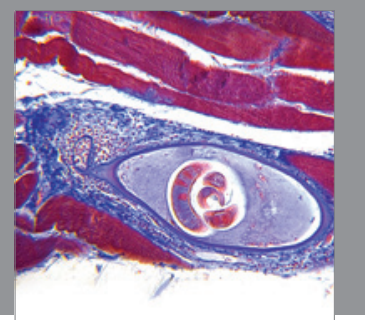

Gastroenterology

Research and Practice
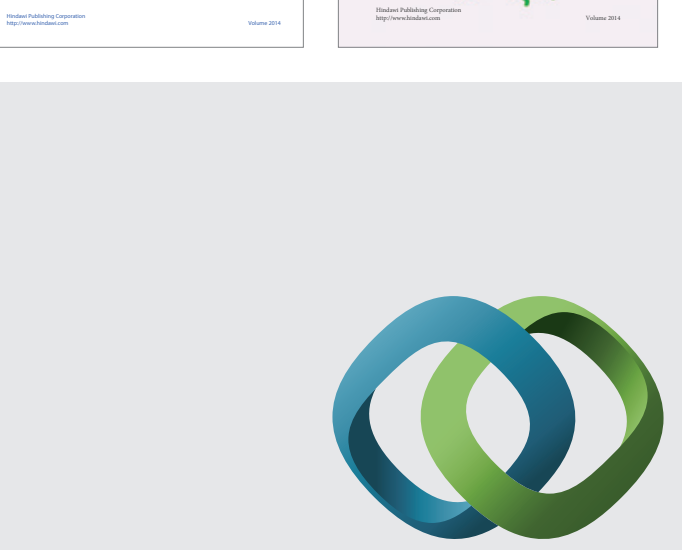

\section{Hindawi}

Submit your manuscripts at

http://www.hindawi.com
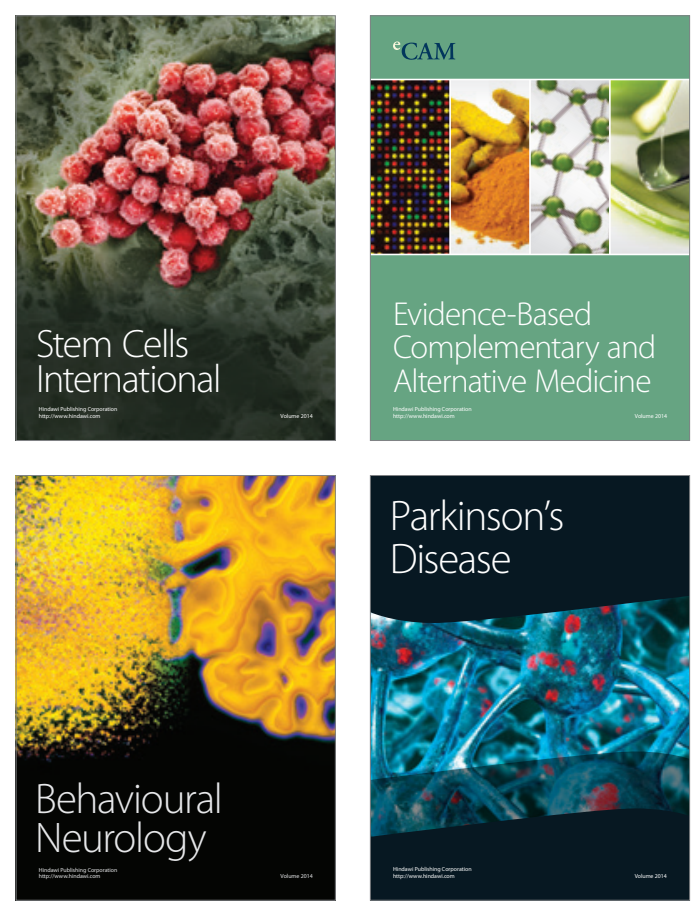

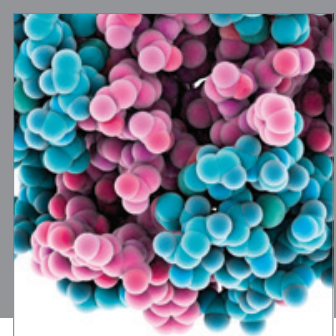

Journal of
Diabetes Research

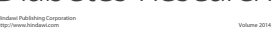

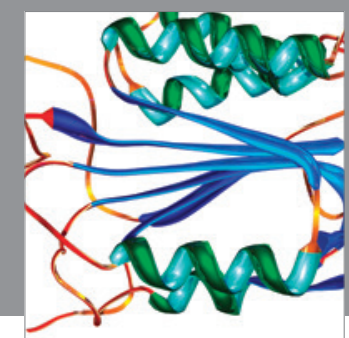

Disease Markers
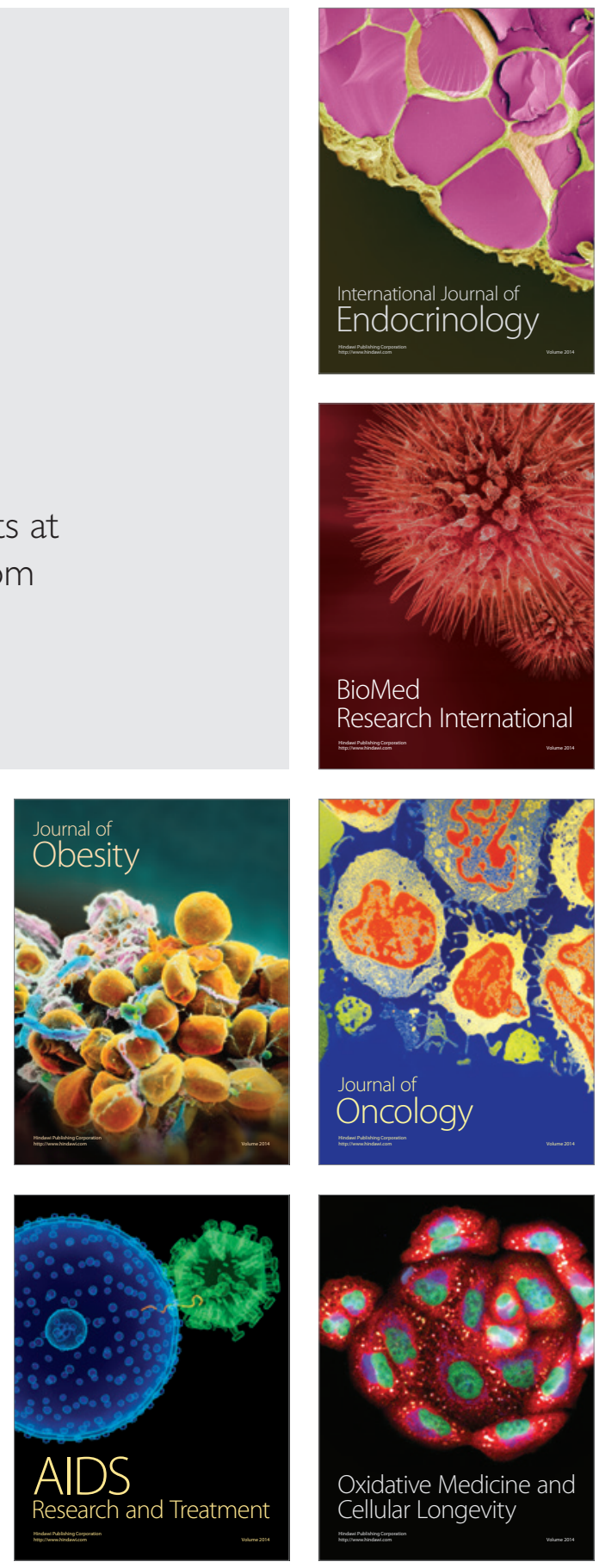